\title{
Fatty acid-induced gut-brain signaling attenuates neural and behavioral effects of sad emotion in humans
}

\author{
Lukas Van Oudenhove,,$^{1,2}$ Shane McKie, ${ }^{3}$ Daniel Lassman, ${ }^{2}$ Bilal Uddin, ${ }^{2}$ Peter Paine, ${ }^{2}$ \\ Steven Coen, ${ }^{4}$ Lloyd Gregory, ${ }^{2}$ Jan Tack, ${ }^{1}$ and Qasim Aziz²,4 \\ ${ }^{1}$ Translational Research Centre for Gastrointestinal Disorders, University of Leuven, Leuven, Belgium. ${ }^{2}$ Department of Gastrointestinal Sciences and \\ ${ }^{3}$ Neuroscience and Psychiatry Unit, University of Manchester, Manchester, United Kingdom. ${ }^{4}$ Wingate Institute for Neurogastroenterology, \\ Queen Mary University, London, United Kingdom.
}

\begin{abstract}
Although a relationship between emotional state and feeding behavior is known to exist, the interactions between signaling initiated by stimuli in the gut and exteroceptively generated emotions remain incompletely understood. Here, we investigated the interaction between nutrient-induced gut-brain signaling and sad emotion induced by musical and visual cues at the behavioral and neural level in healthy nonobese subjects undergoing functional magnetic resonance imaging. Subjects received an intragastric infusion of fatty acid solution or saline during neutral or sad emotion induction and rated sensations of hunger, fullness, and mood. We found an interaction between fatty acid infusion and emotion induction both in the behavioral readouts (hunger, mood) and at the level of neural activity in multiple pre-hypothesized regions of interest. Specifically, the behavioral and neural responses to sad emotion induction were attenuated by fatty acid infusion. These findings increase our understanding of the interplay among emotions, hunger, food intake, and meal-induced sensations in health, which may have important implications for a wide range of disorders, including obesity, eating disorders, and depression.
\end{abstract}

\section{Introduction}

An intimate relationship between hunger/satiety, food intake, visceral sensory signaling, and emotions exists (1). First, feeding supplies essential nutrients, making it critical for survival. Hence, feeding regulatory mechanisms are firmly rooted in homeostasis, giving feeding its intrinsic rewarding nature $(2,3)$. Neurohumoral hunger/satiety signals from the gut to the brain play a crucial role in these homeostatic aspects of feeding $(2,4)$ by continuously signaling the physiological state of the body to the brain $(3,5)$. In Craig's view, "homeostatic emotions," consisting of a feeling and a related motivation, arise from this interoceptive system (3). Second, this intrinsic (homeostatic) reward value of food is strongly modulated by concurrent processing of exteroceptive food-related sensory stimuli (taste, smell, texture, sight) and associated cognitive-affective processes $(2,4,6,7)$. This integrative process determines the overall hedonic experience (subjective pleasantness) associated with feeding, and knowledge of the underlying brain mechanisms is increasing $(2,4,6,7)$. Finally, clinical evidence suggests an important overlap of digestive symptoms, disorders of food intake, and affective symptoms in disorders, including obesity (8), eating disorders (9), functional gastrointestinal disorders (10), and depression (11).

Most neuroimaging research on the link between feeding and emotions has been performed using taste or olfactory stimuli (7), focusing on the integration of interoceptive hunger/satiety signals and exteroceptive sensory signals underlying food-related hedonic experience. Studies on the relationship between "purely interoceptive" homeostatic gut-brain signaling and emotion, on the contrary, are sparse. Our recent study identified a lipid-induced brain

Conflict of interest: The authors have declared that no conflict of interest exists. Citation for this article: J Clin Invest. 2011;121(8):3094-3099. doi:10.1172/JCI46380. activation matrix, using functional magnetic resonance imaging (fMRI) during blinded intragastric infusion of fatty acid compared with that of saline, thereby eliminating exteroceptive food-related stimuli and associated cognitive-affective processes. Activations were found in brainstem, hypothalamus, caudate, thalamus, cerebellum, and mid-cingulate cortex (MCC); these were blocked by the cholecystokinin (CCK) antagonist dexloxiglumide, demonstrating a key role of this gut peptide in lipid-induced gut-brain signaling (12). No differences in hunger, fullness, or nausea sensations between saline or lipid were reported. However, this study did not investigate any putative link with emotion.

The aim of the present study was to investigate a putative interaction between fatty acid-induced gut-brain signaling and experimentally induced sad emotion at both the behavioral and brain level in healthy volunteers. At the brain level, we hypothesized that a fatty acid-by-emotion interaction effect would be found in a number of pre-hypothesized regions of interest (ROIs), including medulla/pons, midbrain, hypothalamus, thalamus, striatum, cerebellum, insula, hippocampus, amygdala, and cingulate cortex (ACC, anterior cingulate cortex; MCC, and posterior cingulate cortex [PCC]). These ROIs were chosen based on our previous study on fatty acid-induced gut-brain signaling (12) and a recent metaanalysis on neuroimaging studies of emotion (13).

\section{Results and Discussion}

Participants underwent four 40-minute fMRI scans on separate days, in randomized order. After a 10-minute baseline period, participants received an intragastric infusion of either fatty acid or saline vehicle and either neutral or sad emotion induction, which started 3 minutes before the intragastric infusion. Ratings of fullness, hunger, nausea, and mood were recorded before the start of scanning and at 3 different time points during scanning (Supple- 
A
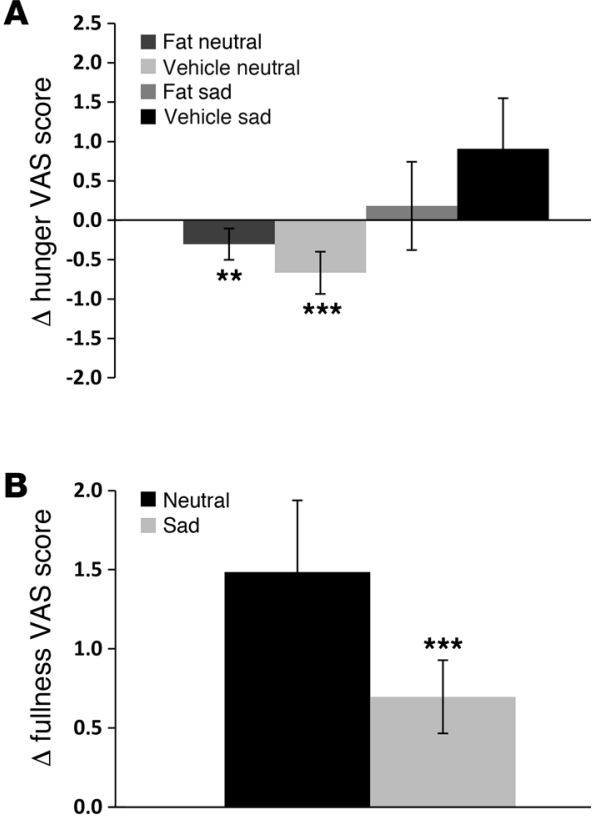

C

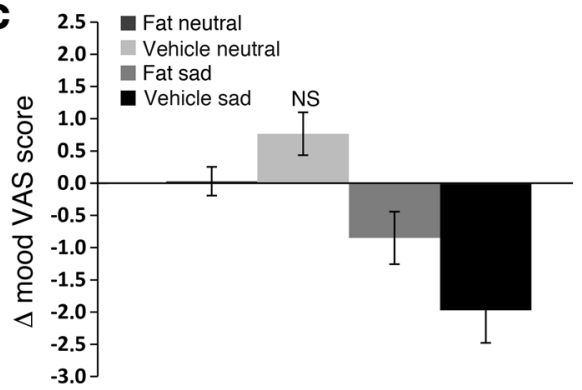

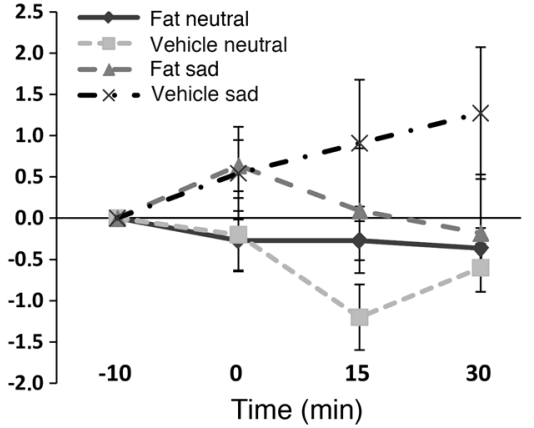
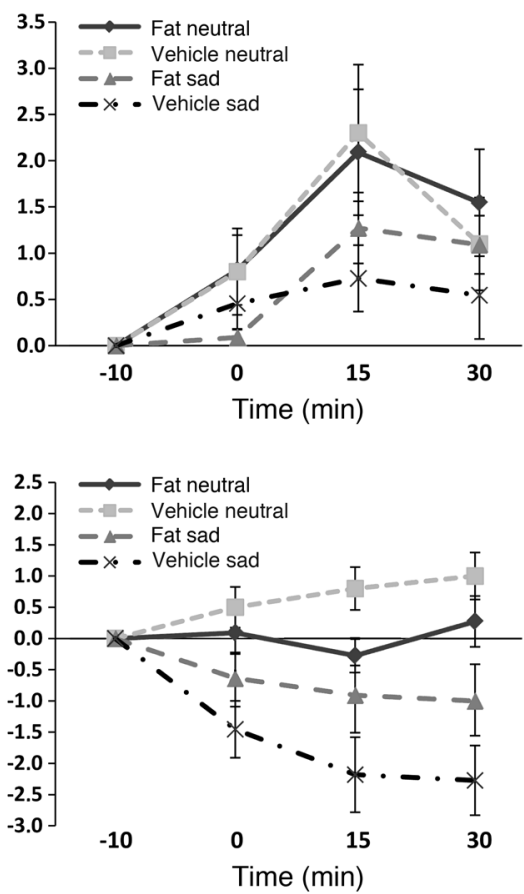

\section{Figure 1}

Effect of sad emotion and intragastric fatty acid on behavioral ratings. (A) Hunger. A significant main effect of emotion (increase of hunger during sad, decrease during neutral) and a significant fat-by-emotion interaction (effect of sad on hunger attenuated by fat) was found. ${ }^{* *} P<0.01$ compared with saline vehicle sad; ${ }^{* * *} P<0.001 \mathrm{com}$ pared with vehicle sad, corrected for multiple comparisons. (B) Fullness. A significant main effect of emotion (smaller increase in fullness during sad compared with neutral) was found. ${ }^{* *} P<0.001$ compared with neutral. (C) Mood. A significant main effect of emotion and a significant fat-by-emotion interaction (attenuation of the effect of emotion induction by fat) was found. All pairwise differences were significant after correction for multiple comparisons, except for vehicle neutral compared with fat neutral (NS, $P=0.13)$. VAS, visual analog scale. mental Figure 1; supplemental material available online with this article; doi:10.1172/JCI46380DS1).

\section{Behavioral}

Hunger ratings. The main effect of emotion $\left[\mathrm{F}_{1,116}=14.44\right.$, $P=0.0002]$ and the fat-by-emotion interaction effect $\left[\mathrm{F}_{1,116}=4.24\right.$, $P=0.04]$ were significant; the main effect of fat was not $\left[\mathrm{F}_{1,116}=\right.$

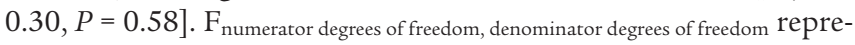
sents the F-statistic from the linear mixed model used to analyze behavioral data (see Methods). As shown in Figure 1A, hunger scores increased during sad emotion and decreased during neutral emotion conditions (main effect of emotion). Furthermore, the difference in hunger ratings between the emotions was much smaller in fat compared with that in saline vehicle conditions (fatby-emotion interaction effect), indicating that the effect of sad mood on hunger is attenuated by fatty acid. The main effect of emotion is in agreement with a recent study in which sad emotion increased the attentional bias for food cues and self-reported hunger/urge to eat in healthy volunteers (14). An increase in the reward value of food cues has been proposed as the cognitive mechanism underlying this relationship and is described in both healthy controls and in patients with obesity and eating disorders $(14,15)$.
Fullness ratings. The main effect of emotion $\left[\mathrm{F}_{1,115}=12.11\right.$, $P=0.0007]$ was significant, contrary to the main effect of fat $\left[\mathrm{F}_{1,115}=0.53, P=0.47\right]$ and the fat-by-emotion interaction effect $\left[\mathrm{F}_{1,115}=0.16, P=0.69\right]$. As shown in Figure $1 \mathrm{~B}$, sad emotion was associated with a smaller increase in fullness compared with neutral emotion. This suggests that reduced perception of fullness may be a mechanism underlying the consumption of larger quantities of food in sad emotional states.

Mood ratings. A significant main effect of emotion was found $\left[\mathrm{F}_{1,116}=60.01, P<0.0001\right]$, confirming the efficacy of the emotion induction procedure, as well as a significant fat-by-emotion interaction effect $\left[\mathrm{F}_{1,116}=15.86, P=0.0001\right]$. The main effect of fat was not significant $\left[\mathrm{F}_{1,116}=0.67, P=0.41\right]$. Figure $1 \mathrm{C}$ shows a decrease in mood ratings during the sad conditions, with no change or a slight increase during the neutral conditions (main effect of emotion). The difference between emotions was smaller in the fat than in the saline vehicle conditions (fat-by-emotion interaction effect), which is consistent with an attenuation of the effect of emotion induction by fatty acid. In humans, evidence points toward a mood-improving role for high-carbohydrate food, with evidence for fatty foods being less conclusive (1). However, the time scale of these studies (days to weeks) is fundamentally different from 
Table 1

Fat-by-emotion interaction effect in pre-hypothesized ROls

\begin{tabular}{|c|c|c|c|c|c|c|c|c|}
\hline Region & $\begin{array}{l}\text { Brodmann } \\
\text { area/subregion }\end{array}$ & Side & $\begin{array}{c}x \\
(\mathrm{~mm})\end{array}$ & $\begin{array}{c}y \\
(\mathrm{~mm})\end{array}$ & $\begin{array}{c}Z \\
(\mathrm{~mm})\end{array}$ & $\begin{array}{l}\text { Cluster } \\
\text { volume }\end{array}$ & $t$-value & $z$-score \\
\hline Brainstem & $\begin{array}{l}\text { Medulla/caudal pons } \\
\text { Midbrain/rostral pons }\end{array}$ & $\begin{array}{l}\text { Left } \\
\text { Right } \\
\text { Left } \\
\text { Right }\end{array}$ & $\begin{array}{c}-9 \\
9 \\
-2 \\
4\end{array}$ & $\begin{array}{l}-38 \\
-36 \\
-32 \\
-26\end{array}$ & $\begin{array}{l}-42 \\
-42 \\
-14 \\
-18\end{array}$ & $\begin{array}{l}100 \\
161 \\
100 \\
170\end{array}$ & $\begin{array}{l}3.99 \\
5.32 \\
3.41 \\
4.11\end{array}$ & $\begin{array}{l}3.88 \\
5.08 \\
3.34 \\
4.00\end{array}$ \\
\hline Hypothalamus & - & $\begin{array}{l}\text { Left } \\
\text { Right }\end{array}$ & $\begin{array}{c}-4 \\
0\end{array}$ & $\begin{array}{l}-4 \\
-2\end{array}$ & $\begin{array}{c}0 \\
-4\end{array}$ & $\begin{array}{c}49 \\
8\end{array}$ & $\begin{array}{l}4.88 \\
3.24\end{array}$ & $\begin{array}{l}4.69 \\
3.18\end{array}$ \\
\hline Caudate head & - & Left & -4 & 8 & 0 & 30 & 3.16 & 3.10 \\
\hline Nucleus accumbens & - & Left & -6 & 8 & -4 & 17 & 3.09 & 3.04 \\
\hline Putamen & - & $\begin{array}{l}\text { Left } \\
\text { Right }\end{array}$ & $\begin{array}{c}-21 \\
28\end{array}$ & $\begin{array}{l}8 \\
0\end{array}$ & $\begin{array}{l}4 \\
0\end{array}$ & $\begin{array}{c}111 \\
22\end{array}$ & $\begin{array}{l}3.57 \\
3.50\end{array}$ & $\begin{array}{l}3.49 \\
3.43\end{array}$ \\
\hline Thalamus & $\begin{array}{l}\text { Ventral lateral nucleus } \\
\text { Pulvinar } \\
\text { Ventral lateral nucleus } \\
\text { Pulvinar }\end{array}$ & $\begin{array}{l}\text { Left } \\
\text { Left } \\
\text { Right } \\
\text { Right }\end{array}$ & $\begin{array}{c}-15 \\
-17 \\
13 \\
23\end{array}$ & $\begin{array}{l}-13 \\
-24 \\
-17 \\
-28\end{array}$ & $\begin{array}{c}11 \\
11 \\
14 \\
4\end{array}$ & $\begin{array}{l}304 \\
304 \\
264 \\
264\end{array}$ & $\begin{array}{l}4.40 \\
3.80 \\
4.11 \\
3.88\end{array}$ & $\begin{array}{l}4.26 \\
3.71 \\
3.99 \\
3.78\end{array}$ \\
\hline Cerebellum & $\begin{array}{l}\text { IX } \\
\text { VIla crus } 2 \\
\text { IX } \\
\text { VIla crus } 1 \\
\text { IX } \\
\text { VI }\end{array}$ & $\begin{array}{l}\text { Left } \\
\text { Left } \\
\text { Right } \\
\text { Right } \\
\text { Right } \\
\text { Right } \\
\text { Right }\end{array}$ & $\begin{array}{c}-30 \\
-38 \\
2 \\
38 \\
13 \\
11 \\
26\end{array}$ & $\begin{array}{l}-49 \\
-55 \\
-58 \\
-60 \\
-83 \\
-41 \\
-66\end{array}$ & $\begin{array}{l}-42 \\
-46 \\
-49 \\
-32 \\
-25 \\
-49 \\
-18\end{array}$ & $\begin{array}{l}1,462 \\
1,462 \\
1,693 \\
1,693 \\
1,693 \\
1,693 \\
1,693\end{array}$ & $\begin{array}{l}4.33 \\
4.29 \\
4.36 \\
4.32 \\
4.32 \\
4.22 \\
4.18\end{array}$ & $\begin{array}{l}4.19 \\
4.16 \\
4.22 \\
4.19 \\
4.19 \\
4.09 \\
4.06\end{array}$ \\
\hline Hippocampus & Cornu ammonis & $\begin{array}{l}\text { Right } \\
\text { Right }\end{array}$ & $\begin{array}{l}39 \\
30\end{array}$ & $\begin{array}{l}-17 \\
-32\end{array}$ & $\begin{array}{l}-21 \\
-4\end{array}$ & $\begin{array}{l}58 \\
71\end{array}$ & $\begin{array}{l}3.98 \\
3.50\end{array}$ & $\begin{array}{l}3.87 \\
3.43\end{array}$ \\
\hline Anterior cingulate & 32 & Left & -11 & 30 & 28 & 21 & 4.01 & 3.90 \\
\hline Mid-cingulate & $\begin{array}{l}23 \\
23 / 24\end{array}$ & $\begin{array}{l}\text { Midline } \\
\text { Midline }\end{array}$ & $\begin{array}{c}2 \\
-6\end{array}$ & $\begin{array}{c}-19 \\
0\end{array}$ & $\begin{array}{l}32 \\
32\end{array}$ & $\begin{array}{c}234 \\
48\end{array}$ & $\begin{array}{l}4.84 \\
4.06\end{array}$ & $\begin{array}{l}4.66 \\
3.95\end{array}$ \\
\hline Posterior cingulate & $\begin{array}{l}29 / 30 \\
23 / 26 \\
29 \\
26\end{array}$ & $\begin{array}{l}\text { Left } \\
\text { Left } \\
\text { Right } \\
\text { Right }\end{array}$ & $\begin{array}{l}-9 \\
-6 \\
11 \\
8\end{array}$ & $\begin{array}{l}-49 \\
-39 \\
-41 \\
-43\end{array}$ & $\begin{array}{l}18 \\
25 \\
11 \\
25\end{array}$ & $\begin{array}{c}56 \\
56 \\
121 \\
121\end{array}$ & $\begin{array}{l}3.41 \\
3.10 \\
4.57 \\
3.88\end{array}$ & $\begin{array}{l}3.34 \\
3.05 \\
4.41 \\
3.78\end{array}$ \\
\hline
\end{tabular}

$t$-values and equivalent $z$-scores are from the peak voxel in each ROI. Cluster volume was measured in voxels. Montreal Neurological Institute coordinates " $x$," " $y$,", and " $z$ " are shown. All clusters are significant at a height threshold of $P_{\mathrm{FDR} \text {-corrected }}<0.05$; no significant effects were found in the insula and amygdala ROIs.

that of the present study, in which a small amount of fatty acid is administered over 2 minutes (1). Further, the majority of previous studies, unlike the present study, have been conducted using consciously perceived food-related stimuli or actual consumption or choice of food (1), rendering it impossible to disentangle the effect of different aspects of feeding (interoceptive-homeostatic, exteroceptive-hedonic, cognitive) on emotion.

Nausea ratings. Virtually no nausea was reported.

Additional results on sex differences can be found in the supplemental material.

\section{fMRI}

As shown in Table 1 and Figure 2, a significant fat-by-emotion interaction effect on the blood oxygen level-dependent signal (BOLD signal) was found in most pre-hypothesized ROIs, including medulla/pons, midbrain, hypothalamus, thalamus, caudate head, putamen, cerebellum, right hippocampus, left pACC (pregenual anterior cingulate cortex), MCC, and PCC. No significant effect was found in the insula or amygdala. Figure 3 shows plots of the BOLD signal change per condition over time in selected ROIs as well as bar graphs of the corresponding AUCs. Including behavioral mood ratings as a covariate in the brain imaging analysis did not substantially change the results (data not shown).

In the neutral emotion conditions, fatty acid infusion was associated with an activation of these brain regions compared with saline vehicle infusion (main effect of fat within neutral emotion), confirming our earlier results (12). In the saline vehicle infusion conditions, sad emotion was associated with a similar activation in these brain areas (main effect of emotion within saline vehicle). This is in line with recent evidence emphasizing that "emotional" brain circuits play a role in the processing of and response to salient events in general rather than to events of a specific affective valence $(13,16)$.

However, most importantly, fatty acid attenuates the effect of sad emotion compared with saline (or sad emotion attenuates the effect of fatty acid compared with that of saline) (fat-by-emotion interaction effect). The pattern was similar in the other ROIs, in which a similar interaction effect was found (data not shown). The neurochemical mechanisms underlying this phenomenon as well 


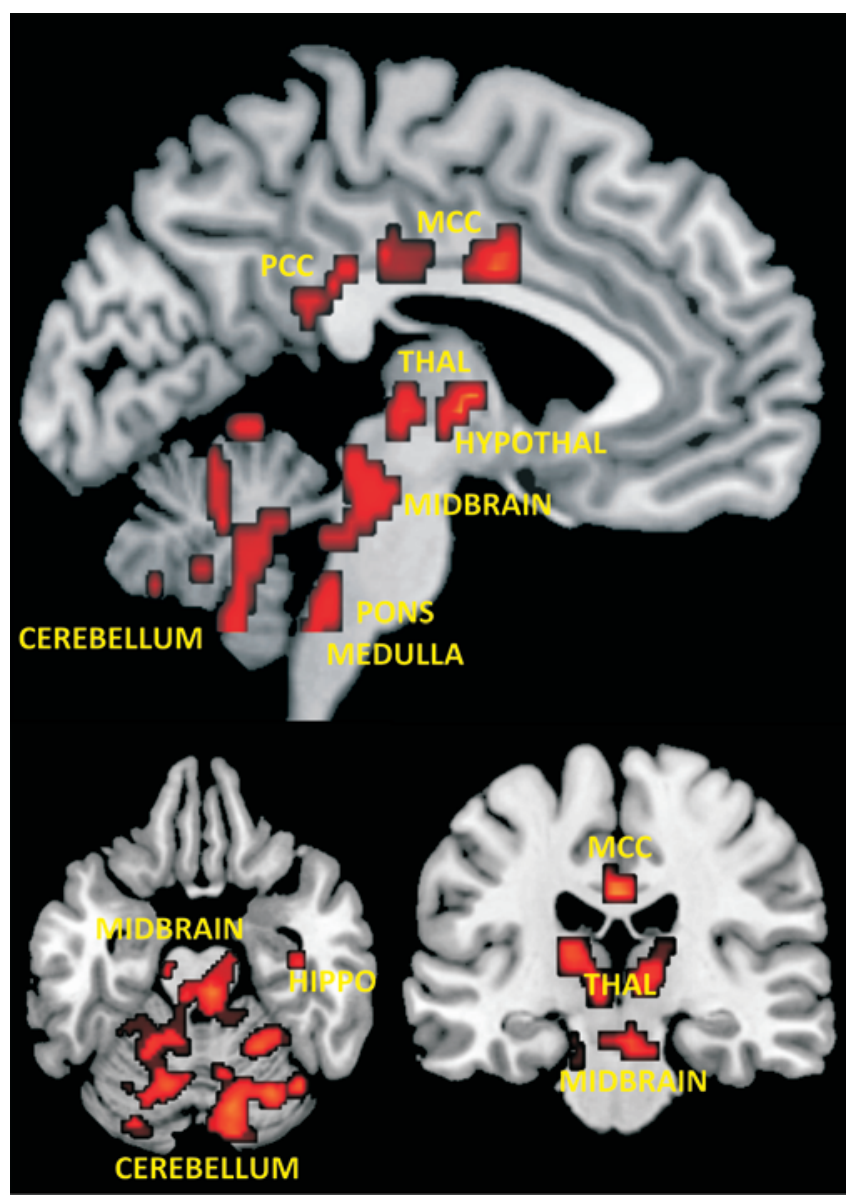

Figure 2

Pre-hypothesized brain ROls in which a significant fat-by-emotion interaction effect was found. Analysis thresholded at $P_{\text {uncorrected }}<0.005$ for display purposes. HYPOTHAL, hypothalamus; THAL, thalamus; HIPPO, hippocampus.

as its physiological function remain to be elucidated, which is a limitation of this study. However, the timing (a few minutes after lipid infusion) and location of the brain responses, in our previous as well as the present study, suggest that gut-brain signaling occurs mainly via the neural route through CCK-induced activation of vagal afferents, although humoral signaling by CCK and other gut peptides cannot be ruled out, as gut hormone levels were not determined in the present study. Despite these limitations, the present results may provide a neurobiological explanation for well-known clinical phenomena, such as "emotional overeating," "comfort feeding," or appetite disturbances in mood disorders. The present results are also in agreement with the view of emotion presented by Damasio et al., as well as with Craig's view on homeostatic emotions (3), in which brain processing of bodily signals in regions included in this study play a pivotal role in the generation and regulation of emotions (17). Additional results on sex differences as well as discussion on a putative differential effect of fatty acid and vehicle on gastric emptying and interoceptive sensitivity can be found in the supplemental material.

In conclusion, we demonstrated, for what we believe to be the first time in humans, that a purely interoceptive, subliminal appe- titive stimulus (intragastric fatty acid infusion in the fasted state) interacts with an exteroceptively generated negative emotional state, at both the behavioral and neural level. More specifically, fatty acid infusion attenuated both the behavioral and neural responses to sad emotion induction. Although the mechanisms underlying this interaction remain to be elucidated, our results may have important implications for understanding the interplay among emotions, hunger, food intake, and meal-induced sensations in health and disorders such as obesity, eating disorders, functional dyspepsia, and depression.

\section{Methods}

\section{Subjects}

Twelve nonobese healthy volunteers were studied in the morning after a 12-hour fast; all gave written informed consent before inclusion. Subjects were blinded to the nature of the intragastric infusion throughout the study; they were not informed about the valence of the emotion induction (neutral or sad) prior to the start of the procedure. The order of conditions was partially counterbalanced using a Latin square design. The study had been given prior approval by the Wrightington, Wigan \& Leigh Local Research Ethical Committee, reference number 06/Q1410/111 (Wigan, United Kingdom). Additional information on the subject recruitment can be found in the supplemental material.

\section{Intragastric nutrient infusion}

A gastric feeding tube was positioned with its tip in the stomach $60 \mathrm{~cm}$ from the incisors and fixed with adhesive tape to the subject's face; this was tolerated well by all subjects. Subjects received $250 \mathrm{ml} 0.05 \mathrm{~mol} / \mathrm{l}$ dodecanoic acid (C12) (Sigma-Aldrich) or $0.9 \%$ saline through the feeding tube over 2 minutes at body temperature, flushed through with $50 \mathrm{ml}$ of water. Solutions were prepared as described previously (12). Additional information on the intragastric nutrient infusion can be found in the supplemental material.

\section{Emotion induction}

Sad or neutral emotion was induced with 2 previously validated methods that were used concurrently to increase the efficacy of emotional modulation. Eleven validated pieces of sad or neutral classical music of 1-minute duration each (18) were played through headphones in randomized order during the 33-minute emotion induction period, which started 3 minutes before the start of the intragastric infusion. At the same time, 10 validated facial expressions depicting either sad or neutral emotion (19) (of the same valence as the music pieces) were back-projected onto a screen visible to the participant by a mirror for 5 seconds each and repeated in random order, only to be interrupted briefly for obtaining ratings.

\section{Sensation and emotion ratings}

At 4 fixed time points during scanning, participants were asked to indicate their sensations of hunger, fullness, and nausea using a 9-point scale with numerical but no verbal anchors. At the same time points, they were asked to rate their mood using a 9-point scale with verbal but no numerical anchors, ranging from sad (indicated by 1 ) to neutral (indicated by 5 ) to happy (indicated by 9). Participants rated each sensation (presented in randomized order) by moving a pointer along the scale, using a response box held in their right hand. Additional information on the MR acquisition can be found in the supplemental material.

\section{Statistics}

All data are shown as mean \pm SEM. $P<0.05$ is considered significant. 


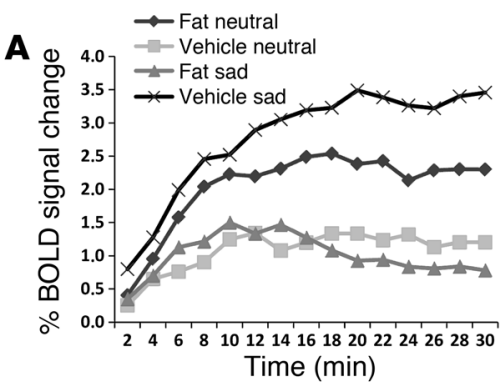

B

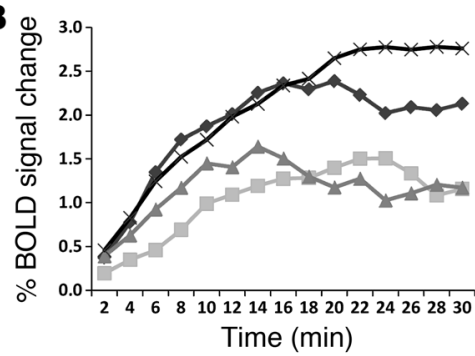

C

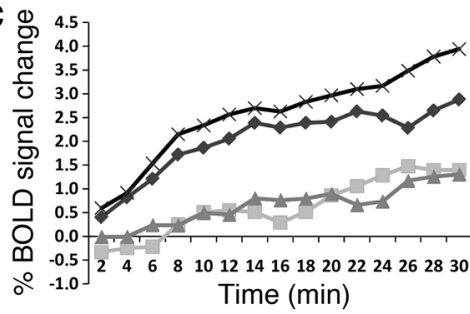

D

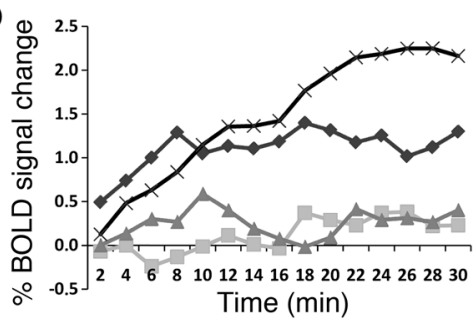

E

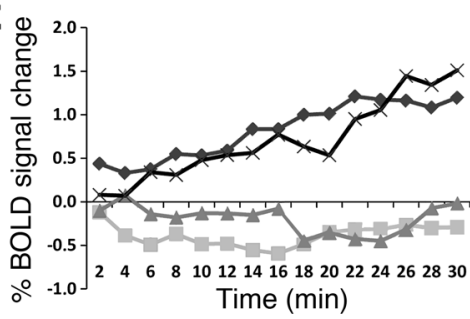

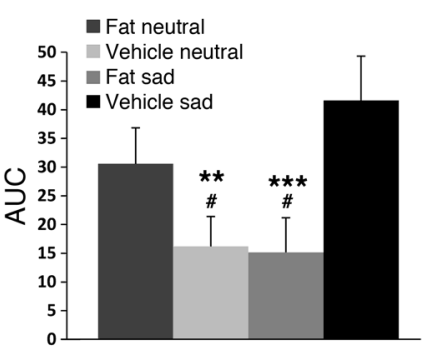
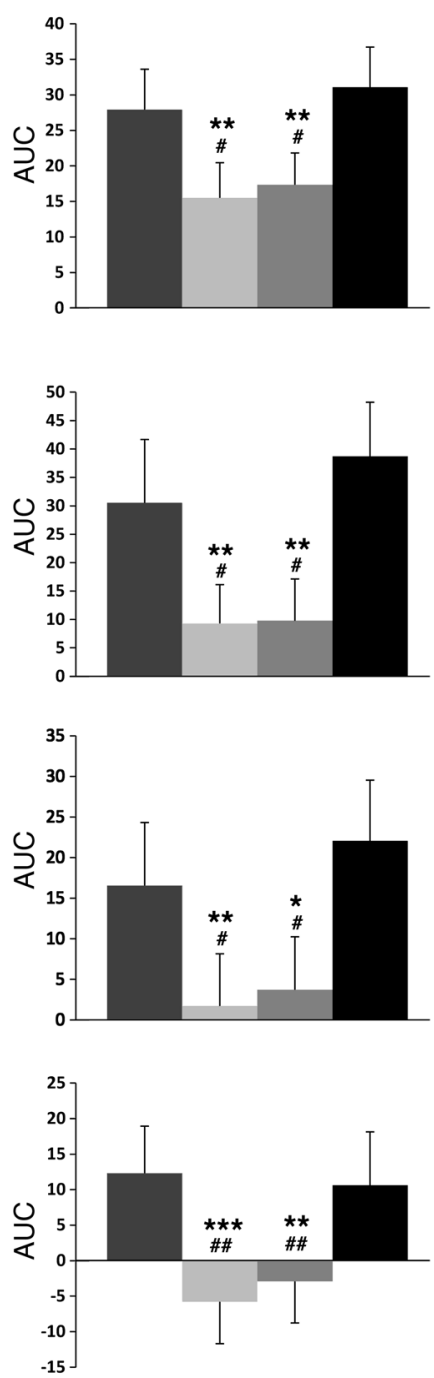

Figure 3

Average time course (percentage BOLD signal change from baseline per 2-minute time bin) and AUC plots for each condition in selected ROIs. (A) Right medulla/pons, (B) midbrain/pons, (C) left hypothalamus, (D) right hippocampus, and $(\mathrm{E})$ MCC. ${ }^{*} P<0.05,{ }^{* *} P<0.01,{ }^{* *} P<0.001$, versus fat neutral; $\# P<0.001,{ }^{\#} P<0.01$ versus saline sad. A significant fat-by-emotion interaction effect on the BOLD signal was found: fatty acid attenuates the effect of sad emotion compared with saline.

and smoothed to facilitate intersubject averaging. One subject was excluded from the analysis because of technical problems during scanning in one visit.

First-level analysis was performed on each participant for each condition in the following ways. The preinfusion and postinfusion volumes were split into time bins; the postinfusion volumes were divided into 15 consecutive 2 -minute ( 24 volume) time bins (T1-T15), and the 24 volumes from the 2 minutes immediately before the lipid/saline infusion formed the baseline time bin (T0). For each participant, the signal averages for the 15 postinfusion time bins (T1-T15) were compared with the baseline average (T0) under each condition (fat neutral [FN], vehicle neutral [VN], fat sad [FS], and vehicle sad [VS]), resulting in 15 first-level $\beta$ images per condition, corresponding to the average BOLD signal change from baseline in each successive postinfusion time bin. The fat-by-emotion interaction was investigated by subtraction between conditions ([FN > VN] > [FS > VS]) for each time bin in each participant, resulting in 15 first-level contrast images, corresponding to the difference in BOLD signal change between sad and neutral emotional state, elicited by fatty acid compared with saline.

A second-level full-factorial ANOVA was carried out with time bin (representing the fat-by-emotion interaction across time) as a repeated measures non-independent factor. This interaction effect analysis was thresholded at a false discovery rate-corrected (FDR-corrected) $P$ value of less than 0.05 in a mask consisting of all pre-hypothesized ROIs, created with the PickAtlas Tool (21). Finally, we explored the fat-by-emotion interaction further by plotting and statistically comparing the average AUC signals in 1-cm cubes whose center was located at the local maxima from the second-level analysis of the interaction effect, for each condition, using the MarsBar Toolbox (22).

\section{Acknowledgments}

The authors thank Barry Whitnall, Dave Pollitt, and Neal Sherratt for technical assistance with MRI data acquisition. This study was funded by the Wellcome Trust Translational Imaging Unit and the Research Foundation - Flanders. L. Van Oudenhove is a postdoctoral research fellow of the Research Foundation - Flanders. Q. Aziz was funded by a Medical Research Council Career Establishment Award.

Received for publication January 12, 2011, and accepted in revised form June 1, 2011.

Address correspondence to: Lukas Van Oudenhove, Herestraat 49, bus 701, B-3000 Leuven, Belgium. Phone: 3216330147; Fax: 3216345939; E-mail: lukas.vanoudenhove@med.kuleuven.be. 
1. Gibson EL. Emotional influences on food choice: Sensory, physiological and psychological pathways. Physiol Behav. 2006;89(1):53-61.

2. Saper CB, Chou TC, Elmquist JK. The need to feed: homeostatic and hedonic control of eating. Neuron. 2002;36(2):199-211.

3. Craig AD. How do you feel? Interoception: the sense of the physiological condition of the body. Nat Rev Neurosci. 2002;3(8):655-666.

4. Rolls ET. Understanding the mechanisms of food intake and obesity. Obes Rev. 2007;8 suppl 1:67-72.

5. Zagon A. Does the vagus nerve mediate the sixth sense? Trends Neurosci. 2001;24(11):671-673.

6. Kringelbach ML. The human orbitofrontal cortex: linking reward to hedonic experience. Nat Rev Neu rosci. 2005;6(9):691-702.

7. Rolls ET. Brain mechanisms underlying flavour and appetite. Philos Trans R Soc Lond B Biol Sci. 2006; 361(1471):1123-1136.

8. Vaidya V. Psychosocial aspects of obesity. Adv Psychosom Med. 2006;27:73-85.

9. Fox JRE, Power MJ. Eating disorders and multilevel models of emotion: An integrated model. Clin
Psychol Psychother. 2009;16(4):240-267.

10. Van Oudenhove L, Aziz Q. Recent insights on central processing and psychological processes in functional gastrointestinal disorders. Dig Liver Dis. 2009;41(11):781-787.

11. Andréasson A, Arborelius L, Erlanson-Albertsson C, Lekander M. A putative role for cytokines in the impaired appetite in depression. Brain Behav Immun. 2007;21(2):147-152.

12. Lassman DJ, et al. Defining the role of cholecystokinin in the lipid-induced human brain activation matrix. Gastroenterology. 2010;138(4):1514-1524.

13. Kober $\mathrm{H}$, et al. Functional grouping and corticalsubcortical interactions in emotion: A meta-analysis of neuroimaging studies. NeuroImage. 2008; 42(2): 998-1031.

14. Hepworth R, Mogg K, Brignell C, Bradley BP. Negative mood increases selective attention to food cues and subjective appetite. Appetite. 2010; 54(1):134-142.

15. Spoor STP, Bekker MHJ, Van Strien T, van Heck GL. Relations between negative affect, coping, and emotional eating. Appetite. 2007;48(3):368-376.
16. Horvitz JC. Mesolimbocortical and nigrostriatal dopamine responses to salient non-reward events. Neuroscience. 2000;96(4):651-656.

17. Damasio AR, et al. Subcortical and cortical brain activity during the feeling of self-generated emotions. Nat Neurosci. 2000;3(10):1049-1056.

18. Mitterschiffthaler MT, Fu CHY, Dalton JA, Andrew CM, Williams SCR. A functional MRI study of happy and sad affective states induced by classical music. Hum Brain Mapp. 2007;28(11):1150-1162.

19. Ekman P, Friesen WV. Pictures Of Facial Affect. Palo Alto, California, USA: Consulting Psychologists Press; 1975.

20. McKie S, et al. Neuronal effects of acute citalopram detected by pharmacoMRI. Psychopharmacology. 2005;180(4):680-686.

21. Maldjian JA, Laurienti PJ, Kraft RA, Burdette JH. An automated method for neuroanatomic and cytoarchitectonic atlas-based interrogation of fMRI data sets. NeuroImage. 2003;19(3):1233-1239.

22. Brett M, Anton J-L, Valabregue R, Poline J-B. Region of interest analysis using an SPM toolbox. NeuroImage. 2002;16:1141-1142. 